\title{
Two Cases of Polypoid Endometriosis: An Uncommon Variant of Endometriosis
}

\author{
Daniela Angerame Yela ${ }^{\mathrm{a}, \mathrm{b}}$, Lucas Trigo ${ }^{\mathrm{a}}$, Helena Giraldo ${ }^{\mathrm{a}}$, Marcelo V Mota ${ }^{\mathrm{a}}$, Liliana L Andrade ${ }^{\mathrm{a}}$, \\ Cristina Laguna Benetti-Pinto ${ }^{\mathrm{a}}$
}

\begin{abstract}
We report two cases of polypoid endometriosis: a 39-year-old woman with menorrhagia and pelvic pain whose ultrasound revealed an intrauterine cystic polypoid mass measuring $4.5 \mathrm{~cm}$ which was removed by hysteroscopy, and a 35-year-old woman with menorrhagia whose ultrasound showed a $3.5 \mathrm{~cm}$ cystic lesion where resection was not possible by hysteroscopy, and the patient underwent hysterectomy. In both, the histological examination confirmed polypoid endometriosis. Polypoid endometriosis is a rare entity that can be found in several places. Here, two cases of polypoid endometriosis in cervical mucosa are described.
\end{abstract}

Keywords: Polypoid endometriosis; Hysteroscopy; Menorrhagia

\section{Introduction}

Polypoid endometriosis is an uncommon variant of endometriosis that is characterized as a lesion which is similar to an endometrial polyp and can be found most often in the colon, ovarian, uterine serosa, cervical canal, vaginal mucosa, ureter, fallopian tube, omentum, bladder peritoneum and paraurethral and paravaginal regions $[1,2]$. We describe two cases of polypoid endometriosis in the cervical canal.

\section{Case Reports}

\section{Case 1}

A 39-year-old woman with four pregnancies, two births and

\footnotetext{
Manuscript accepted for publication December 04, 2014

aDepartment of Gynecology and Obstetrics, School of Medical Sciences, Campinas State University (UNICAMP), Campinas, SP, Brazil

${ }^{\mathrm{b} C}$ Corresponding author: Daniela Angerame Yela, Rua Alexander Fleming, 101, Cidade Universitaria, Campinas, SP, Brazil. Email: yela@unicamp.br
}

doi: http://dx.doi.org/10.14740/jmc2021w two abortions, presented abdominal pain for 1 year, with deep dyspareunia, dysmenorrhea and menorrhagia. She had undergone three previous curettages, one due to an endometrial polyp. Gynecological examination revealed no alterations. The transvaginal ultrasound revealed a $45 \times 24 \times 20 \mathrm{~mm}$ cystic lesion with thick fluid in the endometrial cavity. The patient underwent hysteroscopy which showed a $5 \mathrm{~cm}$ pedunculated lesion in the right lateral wall in the isthmus region containing a cystic area with chocolate colored content (Fig. 1). Resection of the lesion was performed. Histologically, the lesion consisted of smooth muscle cells and showed bleeding inside cysts lined by columnar epithelium; there were endometrial stromal cells immediately below the epithelium (Fig. 2, 3). The diagnosis was polypoid endometriosis.

\section{Case 2}

A diabetic, hypertensive and obese, 35-year-old woman with six pregnancies, five births and one abortion, presented menorrhagia and dysmenorrhea. She used intramuscular medroxyprogesterone acetate with a slight improvement. The transvaginal ultrasound revealed an enlarged uterus $\left(216.1 \mathrm{~cm}^{3}\right)$ and hypoechoic collection in the topography of uterine isthmus measuring $31 \times 35 \times 35 \mathrm{~mm}$, displacing the posterior endometrial cavity; and $12 \times 6 \mathrm{~mm}$ hyperechoic areas with poste-

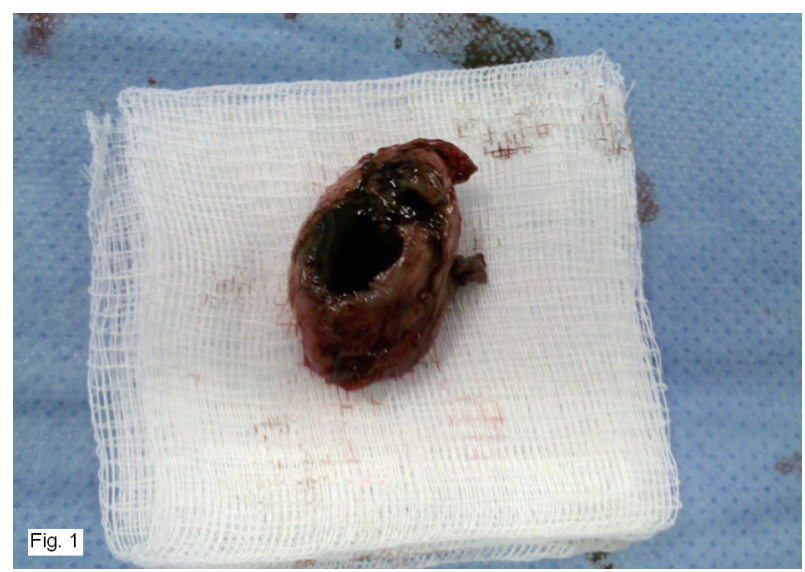

Figure 1. Lesion containing a cystic area with chocolate colored content. 


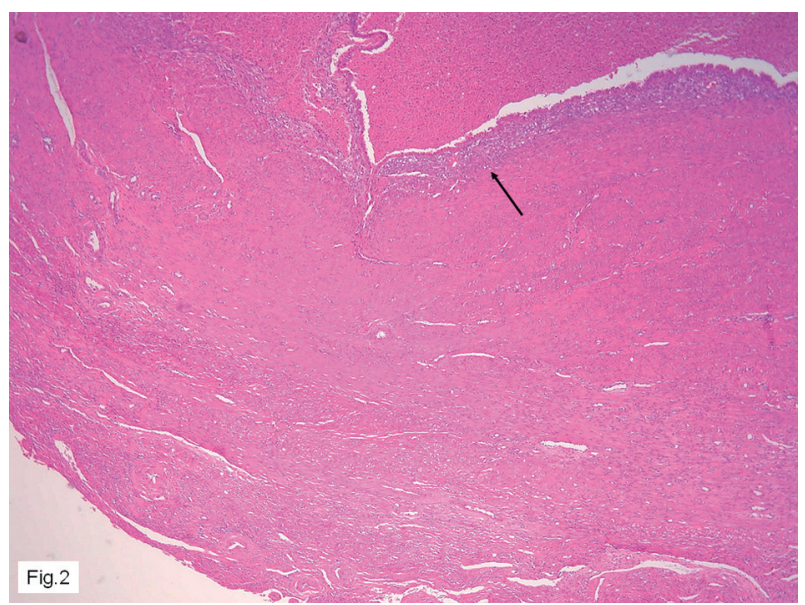

Figure 2. Polypoid lesion consists predominantly of smooth muscle cells, centrally presented with hemorrhagic cystic area content.

rior acoustic shadowing. The patient underwent hysteroscopy which showed a $5 \mathrm{~cm}$ lesion (approximately) in the isthmic anterolateral region. During the resection there was thick chocolate colored output, and the procedure was interrupted due to difficulties.

Histopathological examination revealed fragments of submucosal uterine leiomyoma, epithelioid and hydropic degeneration areas adjacent to the lesion, with intense stromal edema and hemorrhagic areas with morphology suggestive of exogenous hormone-affected endometrium. Immunohistochemistry showed negative HMB45, negative melan, positive 1A4 and positive desmin confirming the diagnosis of leiomyoma with an epithelioid component.

After clinical case discussion, the choice to perform total hysterectomy was made. Histopathological examination revealed a $100 \times 85 \times 60 \mathrm{~cm}$ normal volume uterus, a surface cut showed a $45 \times 35 \times 25 \mathrm{~cm}$ solid well-defined pedicled nodule in the fundus, with small cysts in its interior. Immunohistochemistry revealed positive CD10, positive desmin and positive 1A4 in the mesenchymal component; positive RE in the

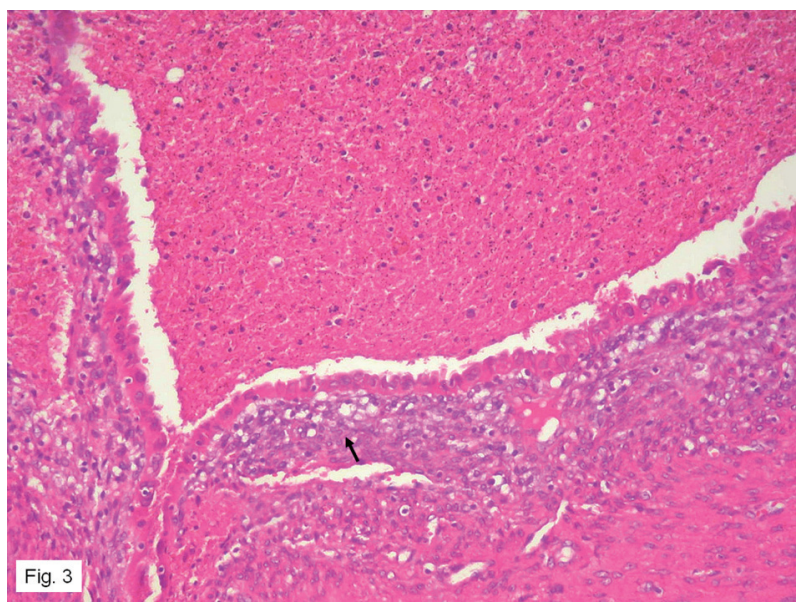

Figure 3. Detail of the cystic area showing endometrial type cylindrical epithelium coating, lined by endometrial stromal cells.

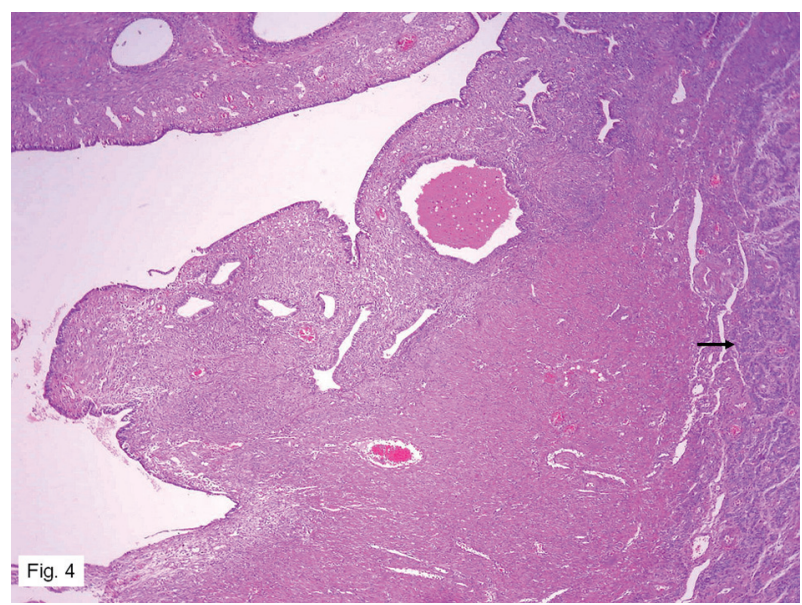

Figure 4. On the surface of the polyp observed dilated endometrial glands and stroma of endometrial type. Note the boundary area between the usual arrangement and arranged smooth muscle fibers cordonal.

mesenchymal cells and glandular epithelium of the lesion and negative inhibin PR. Histologically, there was a pedunculated lesion consisting of apparently normal endometrial glands, stroma and smooth muscle tissue, or epithelioid cordonal focal pattern (Fig. 4, 5). Thick vessels, a characteristic of smooth muscle tumors were observed. An immunohistochemical study of desmin was carried out, which confirmed the smooth muscle nature of the cells with epithelioid appearance. The diagnosis was polypoid endometriosis with a standard focal epithelioid smooth muscle component.

\section{Discussion}

Polypoid endometriosis was first described by Mostoufizadeh and Scully in 1980, for an uncommon and distinctive form of endometriosis with histological features simulating those of a uterine endometrial polyp [3,4]. This form of endometriosis is

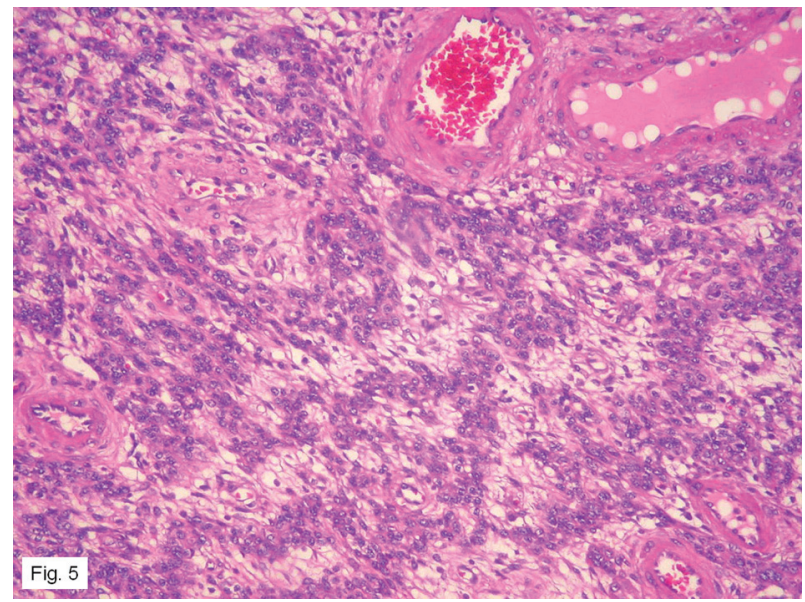

Figure 5. Detail showing the smooth muscle component in cordon epithelioid arrangement. One can notice thick-walled vessels. 
more common in postmenopausal women when compared to traditional endometriosis which is more common in premenopausal women. Its pathogenesis is related in general to the use of hormone therapy (estrogen therapy) or tamoxifen $[1,5]$.

While the clinical manifestations of endometriosis are dysmenorrhea, dyspareunia, chronic pelvic pain and infertility, polypoid endometriosis is related to clinical manifestation such as mass effect [6]. The most common clinical manifestation for polypoid endometriosis, in cervical or vaginal regions, is increased vaginal bleeding, which were found in our cases [1].

This type of endometriosis may form large, often multiple, polypoid masses that not only simulate malignant tumors during surgery, but may also recur after operative removal. However, histopathologic findings resemble endometrial polyps [1]. Polypoid endometriosis was defined as exophytic or polypoid, tumor-like masses that projected from a serosal or mucosal surface or from the lining of an endometriotic cyst. The involvement sites, in order of frequency, include the colon, ovary, uterine serosa, cervical and/or vaginal mucosa, ureter, fallopian tube, omentum, bladder, paraurethral and paravaginal soft tissue and retroperitoneum [1].

Immunohistochemically, glandular ephitelia in polypoid lesions was present in CD10 positive periglandular stroma. This was described in the literature in a 48-year-old woman with cervical polypoid endometriosis [7]. Positive CD10 in immunohistochemical was often found in the endometrial stromal cells and has an important value for the diagnosis of endometriosis [8].

These macroscopic lesions resemble endometrial polyps and can measure from $0.5 \mathrm{~cm}$ to $14 \mathrm{~cm}$ in length and generally are solid lesions with cystic areas [1].

Adenomioma lesions are very little studied and there is a discussion in literature about the origin, i.e., whether they really are neoplasms or represent a variant of adenomyosis, circumscribed in the endometrial cavity. No studies on clonal proliferation of adenomiomas, hindered the concept and the differential diagnosis with adenomyosis. They are also called adenomiomatose polyp and differ from normal endometrial polyps due to a prominent smooth muscle component. Histologically, they consist of three components: endometrial glands, endometrial stroma and smooth muscle cells, with the characteristic presence of caliber and thick blood vessels observed in smooth muscle tumors [9].

Cervical endometriosis is rare and usually found by chance by microscopy. In a Chinese clinic, during 27 years of follow-up, among 13,566 cases of treated endometriosis, there were only 33 cases $(0.2 \%)$ of histologically proven cervical endometriosis [10]. Veiga et al reported an incidence of 0.1$2.4 \%$ in a colposcopic examination series [11].

It is believed that the pathogenesis mechanism of cervical endometriosis occurs through the implantation of endometrial fragments on previously traumatized cervix. Almost $85 \%$ of women have a history of a vaginal delivery or curettage [10]. In our cases the women had a history of abortion with curettage.

The differential diagnosis of this pathology includes several types of tumors as adenofibroma, endometrial stromal sarcoma and adenosarcoma. All these disorders are characterized by benign glands, most commonly proliferative endometrioids. The adenofibromas are characterized by a fibromatous or ovarian type stroma in contrast to the endometrial type stroma of polypoid endometriosis. Adenossarcoma presents at least mild atypical stromal cells and periglandular cellular stromal cuffs, and although the stromal mitotic count may not be particularly helpful in differentiation, a high mitotic count would favor adenosarcoma. Endometrial stromal sarcoma was the characteristic permeative pattern of invasion and frequent vascular invasion $[1,12]$.

Recently, a review of cervical endometriosis that described six cases of cervical polypoid endometriosis was published. In this paper, all cases showed symptoms such as vaginal bleeding, two cases where immunohistochemical was performed, and CD10 and estrogen and progesterone receptors were positive. In this review, four cases were treated with polypectomy, and two were treated with hysterectomy and salpingo-oophorectomy, because there was suspicion of malignancy [12].

The authors described two rare cases of endometriosis that resemble polyps which are usually treated conservatively, but once malignancy is suspected they can be treated in a more interventionist way.

\section{Conflict of Interest}

None declared.

\section{References}

1. Parker RL, Dadmanesh F, Young RH, Clement PB. Polypoid endometriosis: a clinicopathologic analysis of 24 cases and a review of the literature. Am J Surg Pathol. 2004;28(3):285-297.

2. Ozaki k, Gabata T, Tanaka M, Matsui O, Suzuki M, Kawashima $\mathrm{H}$, et al. Polypoid endometriosis: an uncommon and distinctive variant of endometriosis. Eur J Radiol Extra. 2008;65:97-100.

3. Mostoufizadeh M, Scully RE. Malignant tumors arising in endometriosis. Clin Obstet Gynecol. 1980;23(3):951963.

4. Kraft JK, Hughes T. Polypoid endometriosis and other benign gynaecological complications associated with Tamoxifen therapy-a case to illustrate features on magnetic resonance imaging. Clin Radiol. 2006;61(2):198-201.

5. Takeuchi M, Matsuzaki K, Furumoto H, Nishitani H. Case report: A case of polypoid endometriosis: MR pathological correlation. Br J Radiol. 2008;81(964):e118-119.

6. Kaushal S, Dadhwal V, Mathur SR, Ray R, Durgapal P, Deka D. Multifocal polypoid endometriosis in a young woman simulating vaginal and pelvic neoplasm. J Clin Pathol. 2010;63(5):452-454.

7. Felix A, Nogales FF, Arias-Stella J. Polypoid endometriosis of the uterine cervix with Arias-Stella reaction in a patient taking phytoestrogens. Int J Gynecol Pathol. 2010;29(2):185-188. 
8. Sumathi VP, McCluggage WG. CD10 is useful in demonstrating endometrial stroma at ectopic sites and in confirming a diagnosis of endometriosis. J Clin Pathol. 2002;55(5):391-392.

9. Gilks CB, Clement PB, Hart WR, Young RH. Uterine adenomyomas excluding atypical polypoid adenomyomas and adenomyomas of endocervical type: a clinicopathologic study of 30 cases of an underemphasized lesion that may cause diagnostic problems with brief consideration of adenomyomas of other female genital tract sites. Int J Gynecol Pathol. 2000;19(3):195-205.
10. Wang S, Li XC, Lang JH. Cervical endometriosis: clinical character and management experience in a 27-year span. Am J Obstet Gynecol. 2011;205(5):452 e451-455.

11. Veiga-Ferreira MM, Leiman G, Dunbar F, Margolius KA. Cervical endometriosis: facilitated diagnosis by fine needle aspiration cytologic testing. Am J Obstet Gynecol. 1987; 157(4 Pt 1):849-856.

12. Jaiman S, Gundabattula SR, Pochiraju M, Sangireddy JR. Polypoid endometriosis of the cervix: a case report and review of the literature. Arch Gynecol Obstet. 2014;289(4):915-920. 